\title{
Structure and SHG of the high pressure phase IV of $\mathrm{HgBr}_{2}$
}

\author{
Marc Hostettler ${ }^{\mathrm{a}, *}$, Dieter Schwarzenbach ${ }^{\mathrm{b}}$, Jan Helbing ${ }^{\mathrm{c}}$, \\ Vladimir Dmitriev ${ }^{\mathrm{d}}$, Hans-Peter Weber ${ }^{\mathrm{b}, \mathrm{d}}$ \\ ${ }^{\mathrm{a}}$ Laboratorium für chemische und mineralogische Kristallographie, University of Bern, CH-3012 Bern, Switzerland \\ ${ }^{\mathrm{b}}$ Laboratoire de cristallographie, EPFL/SB/IPMC/LCR, Swiss Federal Institute of Technology Lausanne, CH-1015 Lausanne, Switzerland \\ ${ }^{\mathrm{c}}$ Institut de Physique de la Matière Condensée, University of Lausanne, BSP Dorigny, CH-1015 Lausanne, Switzerland \\ ${ }^{\mathrm{d}}$ Swiss Norwegian Beam Lines, ESRF, BP 220, F-38043 Grenoble Cedex, France
}

Received 2 October 2003; accepted 5 November 2003 by P. Wachter

\begin{abstract}
Angle dispersive X-ray diffraction experiments on mercuric bromide $\left(\mathrm{HgBr}_{2}\right)$ under high pressure up to $11.0 \mathrm{GPa}$ were carried out at room temperature using synchrotron radiation. In addition to the already known four different phases of $\mathrm{HgBr}_{2}$ in the pressure-temperature range of $p<4.5 \mathrm{GPa}, 90<T<600 \mathrm{~K}$ our observations show the existence of a new phase (V) above $9.0 \mathrm{GPa}$ and, together with published material, support the phase transition sequence: (I) orthorhombic-(II) orthorhombic-(III) monoclinic-(IV) trigonal-(V) trigonal/hexagonal. The structure of phase IV with space group symmetry $P 3$ has been determined from powder diffraction data. The observation of second-harmonic-generation signals confirms the absence of an inversion center. The structure of phase IV is a commensurately modulated variant of the $\mathrm{CdI}_{2}$ type layer structure, where part of the $\mathrm{Hg}$ atoms are displaced from the centers of the $\mathrm{HgBr}_{6}$ octahedra by a much as $0.76 \AA$ in the direction perpendicular to the layers.
\end{abstract}

(C) 2003 Elsevier Ltd. All rights reserved.

PACS: $61.50 . \mathrm{Ks} ; 81.30 .-\mathrm{t}$; $42.70 . \mathrm{Mp}$

Keywords: E. High pressure; E. Synchrotron radiation; C. Crystal structure and symmetry; D. Phase transitions

\section{Introduction}

The solid phases of the mercuric halides $\mathrm{HgX}_{2}(\mathrm{X}=\mathrm{I}$, $\mathrm{Br}, \mathrm{Cl})$ form two kinds of crystal structures: (i) closest sphere packings of $\mathrm{X}$ with $\mathrm{Hg}$ in tetrahedral interstices; (ii) linear or bent $\mathrm{HgX}_{2}$ molecules resulting in distorted closest packings of $\mathrm{X}$. $\mathrm{HgI}_{2}$ is the most intensively investigated mercuric halide; its red modification is used as roomtemperature detector material for $\mathrm{X}$ - and $\gamma$-rays [1-4]. Besides the stable red phase, $\mathrm{HgI}_{2}$ also forms an orange and a yellow phase at ambient conditions, both of which are metastable [5]. The orange phase comprises three different closely related structures, which may occur together in a

\footnotetext{
* Corresponding author. Tel.: +41-21-631-4274; fax: +41-31631-3996.

E-mail address: marc.hostettler@krist.unibe.ch (M. Hostettler).
}

multiply twinned conglomerate [6]; the structure of the yellow phase [5] is related to the high-temperature yellow phase [7]. The structural motifs of these polymorphs are: corner-shared $\mathrm{HgI}_{4}$ tetrahedra (structure kind i, red), cornershared $\mathrm{Hg}_{4} \mathrm{I}_{10}$ supertetrahedra (i, orange), and $\mathrm{HgI}_{2}$ molecules (ii, yellow).

At ambient conditions, phase $\mathrm{I}$ of $\mathrm{HgBr}_{2}$ adopts the structure of the yellow metastable polymorph of $\mathrm{HgI}_{2}[8,9]$. It is orthorhombic, polar space group symmetry $C m c 2_{1}$ $\left(D_{2 h}\right)$, and thus presents opto-electronic properties; it is also a candidate material for the detection of $\gamma$ - rays [10]. At ambient pressure, phase I is stable from $90 \mathrm{~K}$, the lowest temperature of observation, to the melting temperature of $509 \mathrm{~K}$. The application of pressure up to $4.5 \mathrm{GPa}$ induces several transitions as revealed by dilatometry (compressioncurve method) [11] and Raman spectroscopy [12]. Three phases (II-IV) have been reported in this pressure range 
(Fig. 1), the structures of which have not yet been determined. In order to better understand the behavior of this material under pressure, we performed X-ray powder diffraction and second-harmonic-generation (SHG) experiments using diamond-anvil cells (DAC). In this communication, we present an overview of the diffraction results, the crystal structure of phase IV and its SHG signal, as well as the occurrence of an additional phase $\mathrm{V}$.

\section{Experimental}

\subsection{X-ray diffraction}

In situ high-pressure angle-dispersive $\mathrm{X}$-ray diffraction data were recorded at the Swiss-Norwegian Beam Lines at the European Synchrotron Radiation Facility (ESRF, Grenoble, France) by using monochromatic radiation with wavelength $\lambda=0.7498$ (2) $\AA$ collimated to a diameter of $100 \mu \mathrm{m}$. Commercial $\mathrm{HgBr}_{2}$ powder (Fluka, puriss. p.a. $>99 \%$ ) composed of needle shaped crystallites was ground to an average particle size of $5-10 \mu \mathrm{m}$. The sample was introduced into the hole of a tungsten gasket in a DAC [13] together with a ruby chip and silicone oil as pressure medium. The pressure was determined by the fluorescence of the ruby [14]. Diffraction data were recorded with a MAR345 area detector at several pressures up to $11 \mathrm{GPa}$. The 2D images were corrected for geometrical distortions and transformed into a $1 \mathrm{D}$ powder diagram by integration with program Fit2D [15]. Diffraction intensities were corrected for absorption of the DAC.

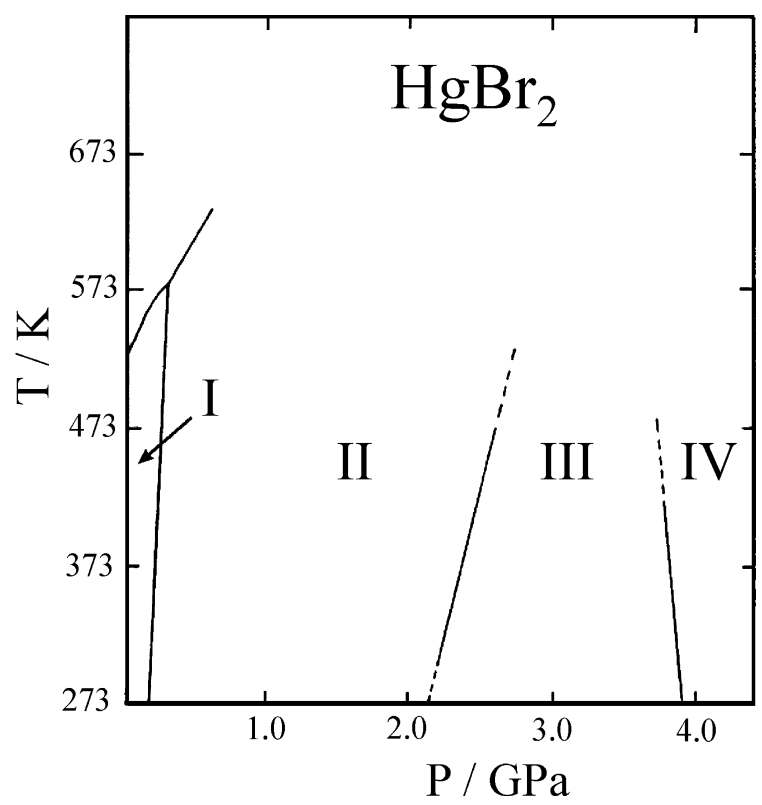

Fig. 1. Phase diagram of $\mathrm{HgBr}_{2}$, the phase boundaries have been established by Raman spectroscopy, adapted from [12].

\subsection{Second-harmonic generation}

The first description of a SHG experiment using powders has been reported in Ref. [16]. We have performed a similar experiment using a femtosecond Ti:sapphire laser at $810 \mathrm{~nm}$. Second-harmonic signals were measured in transmission geometry. Filters and a spectrometer were used to suppress the $810 \mathrm{~nm}$ light, and spectra in the $405 \mathrm{~nm}$ region were recorded with a CCD detector. Powdered sample contained in a capillary was first investigated at ambient conditions (phase I). The same experiment was repeated with a DAC, at a pressure of 6.2(1) GPa (phase IV) and signals were scaled to the respective sample volumes.

\section{Results}

\subsection{Phase transition sequence}

Up to the III-IV transition at $4 \mathrm{GPa}$, the powder rings show the sample to be strongly textured as evidenced by the raw 2D images of Fig. 2. The diagrams of phase II are very similar to those of phase I. Because of the texture, we could not identify any structural change between these two phases. The I-II transition was first detected by dilatometry. It is accompanied by a volume change similar to the one observed for the II-III and III-IV transitions [11]. However, its existence was not conclusively confirmed by Raman spectroscopy, the reported differences between I and II being minor [12]: for I, the observed peaks were at 16, 57 and $186 \mathrm{~cm}^{-1}$, while for II the first peak was absent and the others had shifted by +5 and $-2 \mathrm{~cm}^{-1}$, respectively. Phase III displays a more complicated diffraction pattern, with additional powder lines, suggesting a lower, possibly monoclinic symmetry. However, also for this phase the texture did not permit us to propose a convincing indexation. In addition, the corresponding Raman spectrum shows inferior signal to noise compared to the spectra of phases I, II and IV [12]. These difficulties might be due to the formation of a strongly disordered state in the stability range of phase III of $\mathrm{HgBr}_{2}$. At the III-IV transition the texture disappears. This

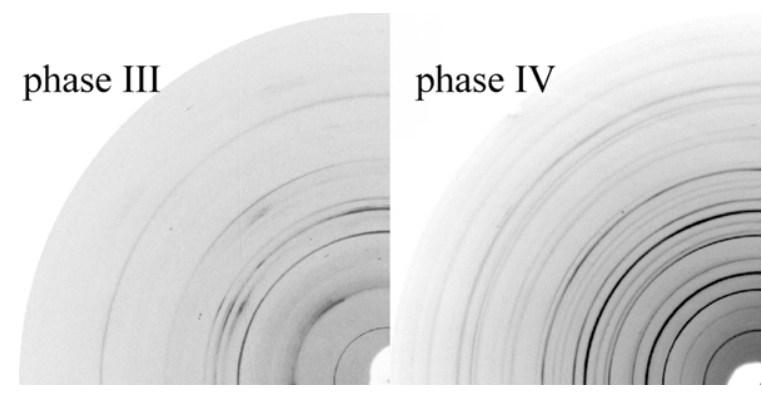

Fig. 2. Raw powder diffraction data collected at 3.4(1) GPa (left, phase III) and 5.4(1) GPa (right, phase IV), illustrating the disappearance of the texture at the III $\rightarrow$ IV transition. 
observation establishes the destructive character of the transition. The powder data of phase IV can be indexed in a hexagonal cell, with $a=6.7569$ (3) and $c=5.6589(9) \AA$ at 5.4(1) GPa. Above $9.0 \mathrm{GPa}$, additional peaks appear, they are ascribed to an additional phase $\mathrm{V}$. The corresponding powder pattern can be indexed with the unit cell of phase IV by doubling the $c$ axis, $a=6.787(1)$ and $c=11.130(2) \AA$ at $10.7 \mathrm{GPa}$.

\subsection{Second-harmonic generation}

Both phases I and IV of $\mathrm{HgBr}_{2}$ generate a clear secondharmonic signal. The observed integrated intensities are scaled with the respective volume of the sample to allow the comparison of the relative conversion efficiencies of the two phases, giving 250,000 and 230,000 photons $\mathrm{s}^{-1} \mathrm{~mm}^{-3}$ for phase I and IV, respectively. These values are of the same order of magnitude as those measured for a powdered sample of the yellow high-temperature phase of $\mathrm{HgI}_{2}$ (space group symmetry $\mathrm{P} 2_{1}$ ), and about three orders of magnitude larger than the value measured for the centrosymmetric red phase of $\mathrm{HgI}_{2}$ at room temperature [7]. Red $\mathrm{HgI}_{2}$ was used as a centrosymmetric reference material to estimate the magnitude of a possible surface effect for our experimental set-up. The structures of phase I of $\mathrm{HgBr}_{2}$ and of the yellow high-temperature phase of $\mathrm{HgI}_{2}$ are known to be noncentrosymmetric and the signal measured for phase IV is too large for a surface effect. Therefore, it is safe to conclude that the inversion is also absent in the structure of phase IV of $\mathrm{HgBr}_{2}$.

\subsection{Structure of phase IV}

The X-ray diffraction intensities do not present any systematic absences. The structure was solved with direct methods [17]. Different hexagonal and trigonal space groups were tested and an initial model was obtained in the noncentrosymmetric space group $P 3$, in agreement with the observed second-harmonic signal. This structure was refined with the Rietveld method using program GSAS [18], leading to the confidence factors: $w R_{\mathrm{p}}=0.035, R_{\mathrm{p}}=$ 0.025 and $R\left(F^{2}\right)=0.075$ [19]. The experimental data, refined profile and their difference curve are shown in Fig. 3. The structural parameters are given in Table 1.

The structure of phase IV of $\mathrm{HgBr}_{2}$ is a derivative of the structure of $\mathrm{CdI}_{2}$. It is built from layers of edge-sharing octahedra that are stacked through $\mathrm{Br} \cdots \mathrm{Br}$ van der Waals contacts. In contrast to the $\mathrm{CdI}_{2}$ type structure, some of the coordinated metal atoms are displaced along $c$ from the centroïds of the octahedra, leading to three distinct mercury sites and thus $Z=3$ instead of $Z=1$. Fig. 4 presents a view of the structure onto the plane of the layer [ $\left[\begin{array}{lll}0 & 0 & 1\end{array}\right]$, and an edge view of the layer down the $\left[\begin{array}{lll}1 & 1 & 0\end{array}\right]$ direction showing the alternating displacements along $\mathbf{c}$ of the three mercury atoms in neighboring octahedra along [ $\left[\begin{array}{lll}1 & 1 & 0\end{array}\right]$. This structure may be described as a commensurate modulation of the $\mathrm{CdI}_{2}$

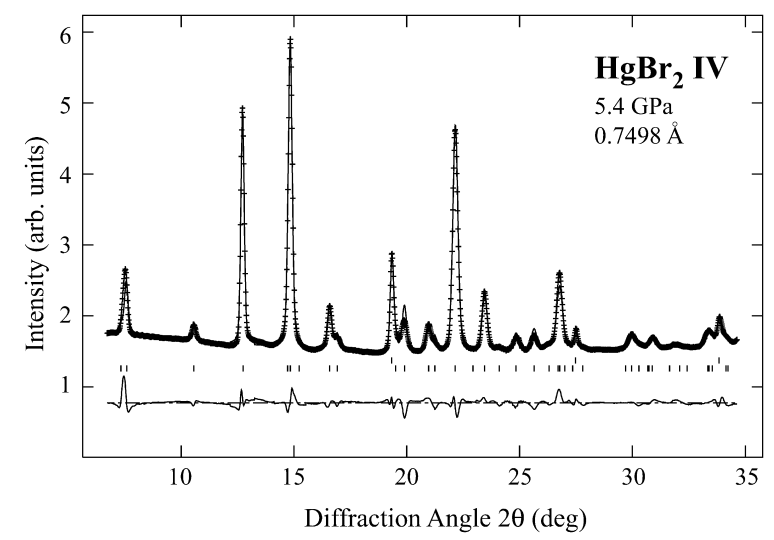

Fig. 3. Diffraction pattern of the phase IV of $\mathrm{HgBr}_{2}$ at $5.4 \mathrm{GPa}$. Experimental and refined profiles are drawn, respectively, with crosses and full line. Their difference is the lower curve. The ticks indicate the peak positions. The contamination by the diffraction of the gasket was modelled with an additional phase.

structure. The $\mathrm{Hg}-\mathrm{Br}$ bond lengths are 2.56(1), 2.60(1) $\AA$ for $\mathrm{Hg} 1,2.60(1), 2.72(1) \AA$ for $\mathrm{Hg} 2$ and 2.63(2), 3.10(1) $\AA$ for $\mathrm{Hg} 3 . \mathrm{Hg} 1, \mathrm{Hg} 2$ and $\mathrm{Hg} 3$ are displaced from the centers of the coordination octahedra by $+0.02,-0.19$ and $+0.76 \AA$, respectively. Thus, $\mathrm{Hg} 3$ adopts a trigonal coordination whereas $\mathrm{Hg} 1$ and $\mathrm{Hg} 2$ show an approximately octahedral coordination with six bonds of roughly the same length. The interlayer $\mathrm{Br} \cdots \mathrm{Br}$ contacts are 3.48(1), 3.64(2) and 3.78(2) $\AA$. Surprisingly, the average isotropic displacement parameter of the $\mathrm{Hg}$ atoms is 1.7 times larger than the one of $\mathrm{Br}$. Even if the atomic displacement parameters derived from powder data are often unrealistic, this observation of larger displacement parameters for the mercury atoms has also been observed for the red and orange $\mathrm{HgI}_{2}$ structures determined from accurate single crystal data $[6,7]$.

\section{Conclusions}

The diffraction experiments have shown the existence of an additional phase (V) of $\mathrm{HgBr}_{2}$ above $9.0 \mathrm{GPa}$. Together with published material $[8,9]$, they suggest a phase transition

Table 1

Structural parameters of phase IV of $\mathrm{HgBr}_{2}$ at 5.4(1) GPa, unit cell dimensions $a=6.7569(3)$ and $c=5.6589(9) \AA$, space group symmetry $P 3, Z=3$. Atomic isotropic displacement parameters are $U_{\text {iso }}(\mathrm{Hg})=0.0336(18), U_{\text {iso }}(\mathrm{Br})=0.0190(17) \AA^{2}$

\begin{tabular}{lllll}
\hline Atom & Wyckoff site & $x$ & $y$ & $z$ \\
\hline $\mathrm{Hg} 1$ & $1 a$ & 0.0 & 0.0 & $0.4699(7)$ \\
$\mathrm{Hg} 2$ & $1 c$ & 0.66667 & 0.33333 & $0.4329(7)$ \\
$\mathrm{Hg} 3$ & $1 b$ & 0.33333 & 0.66667 & $0.6000(11)$ \\
$\mathrm{Br} 1$ & $3 d$ & $0.676(2)$ & $0.698(2)$ & $0.216(2)$ \\
$\mathrm{Br} 2$ & $3 d$ & $0.3470(2)$ & $0.2969(2)$ & $0.7161(7)$ \\
\hline
\end{tabular}




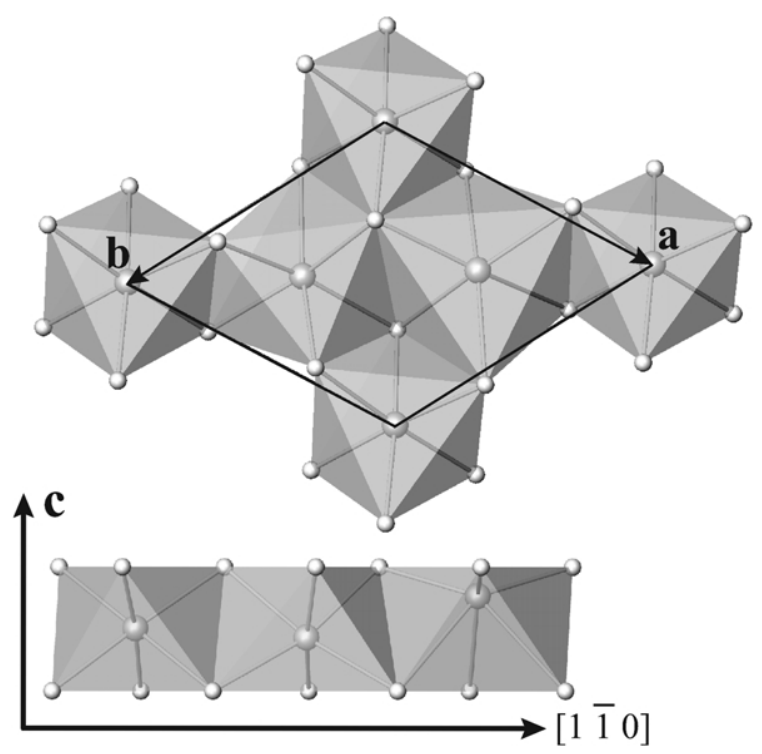

Fig. 4. Structure of the phase IV of $\mathrm{HgBr}_{2}$ at $5.4 \mathrm{GPa}$. Up: view along the trigonal $c$-axis onto the layer of edge-shared octahedra.

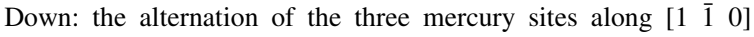
direction.

sequence, (I) orthorhombic-(II) orthorhombic-(III) monoclinic-(IV) trigonal-(V) trigonal or hexagonal. Due to strong texture effects, we cannot unambiguously confirm or disprove the existence of the I-II transformation, and we are unable to index the pattern and ascertain the proposed symmetry of phase III. The destructive character of the III-IV transition considerably reduces the texture effects, allowing a complete structure determination of phase IV. This phase forms a commensurately modulated $\mathrm{CdI}_{2}$ structure built from layers of edge-sharing octahedra, in which one out of three mercury atoms assumes a trigonal rather than an octahedral coordination. This structure and its atomic displacement parameters suggest mercury to be mobile and recall the transformation of orange into red $\mathrm{HgI}_{2}$ where mercury also appears to be mobile inside the iodineatom substructure and diffuses between tetrahedral voids [6]. The structure of phase IV of $\mathrm{HgBr}_{2}$ may explain the shift of the $\mathrm{Hg}-\mathrm{Br}$ Raman frequencies to lower values with increasing pressure [12]. Usually, a vibrational mode shifts to higher frequency upon increasing pressure. The structural reason for the decreasing frequency observed for $\mathrm{HgBr}_{2}$ may be the tendency of mercury to shift to higher coordination with increasing pressure.

The results presented here are a further confirmation that $\mathrm{Hg}$ (II) can adopt a considerable variety of environments in condensed matter, including tetrahedral, trigonal, linear and octahedral coordination. Each coordination may derive from a region of stability in the $p-T$ space associated with particular values of the energy levels of the valenceelectrons. For $\mathrm{HgBr}_{2}$ the low pressure region of the phase diagram seems to favor molecular structures with a coordination number of two, while pressures above 4.0 GPa stabilize pyramidal and octahedral coordination with trigonal and hexagonal symmetries.

The halides of the other group IIB elements $\mathrm{Zn}$ and $\mathrm{Cd}$ form non-molecular structures with closest-packed anions. The structures of the zinc halides are tetrahedral [20] analogous (but not identical) to those of $\mathrm{HgI}_{2}$, whereas the cadmium halides form the well-known octahedral layer structures of which phase IV of $\mathrm{HgBr}_{2}$ is a variant.

\section{Acknowledgements}

We thank Prof. Majed Chergui of the Laboratoire de spectroscopie ultrarapide, Swiss Federal Institute of Technology at Lausanne for providing the setup used in the SHG experiments. We also thank the staff of the SwissNorwegian Beam Lines at the ESRF, Grenoble, for their competent help. This work, as well as in part the SwissNorwegian Beam Lines, has been funded by the Swiss National Science Foundation.

\section{References}

[1] M. Piechotka, Mater. Sci. Eng. 18 (1997) 1.

[2] B. Steiner, L. van dan Berg, U. Laor, J. Appl. Phys. 86 (1999) 4677.

[3] L. Fornaro, L. Luchini, M. Köncke, L. Mussio, E. Quagliata, K. Chattopadhyay, A. Burger, J. Cryst. Growth 217 (2000) 263.

[4] M. Schieber, H. Hermon, A. Vilensky, L. Melekhov, R. Shatunovsky, E. Meerson, H. Saado, Nucl. Instrum. Methods Phys. Res., Sect. A 458 (2001) 41.

[5] G.A. Jeffrey, M. Vlasse, Inorg. Chem. 6 (1967) 396.

[6] D. Schwarzenbach, Z. Kristallogr. 128 (1969) 97. M. Hostettler, H. Birkedal, D. Schwarzenbach, Acta Cryst. B58 (2002) 903. M. Hostettler, D. Schwarzenbach, Acta Cryst. B58 (2002) 914. M. Hostettler. PhD Thesis, Université de Lausanne, Switzerland, 2002.

[7] M. Hostettler, H. Birkedal, D. Schwarzenbach, Helv. Chim. Acta 86 (2003) 1410. M. Hostettler, H. Birkedal, D. Schwarzenbach, Chimia 55 (2001) 541.

[8] H. Braekken, Z. Kristallogr. 83 (1932) 222.

[9] V.I. Pakhomov, A.V. Goryunov, I.N. Ivanova-Korfini, A.A. Boguslavskii, R.Sh. Lotfullin, Russ. J. Inorg. Chem. 35 (1990) 1407.

[10] R. Farrell, F. Olschner, K. Shah, M. Squillante, Nucl. Instrum. Methods Phys. Res., Sect. A 387 (1997) 194

[11] P.W. Bridgman, Proc. Am. Acad. Arts Sci. 72 (1937) 45.

[12] D.M. Adams, R. Appleby, J. Chem. Soc. Dalton (1977) 1535.

[13] R. Miletich, D.R. Allan, W.F. Kuhs, MSA Rev. Mineral. Geochem. 41 (2001) 445.

[14] R. Munro, G. Piermarini, S. Block, W. Holzapfel, J. Appl. Phys. 57 (1985) 165.

[15] A. P. Hammersley, ESRF Internal Report, V9.129 Reference Manual V3.1, 1998. A.P. Hammersley, S.O. Svensson, 
M. Hanfland, A.N. Fitch, D. Hausermann, High Press. Res. 14 1996235.

[16] S.K. Kurtz, T.T. Perry, J. Appl. Phys. 39 (1968) 3798.

[17] A. Altomare, M.C. Burla, G. Cascarano, C. Giacovazzo, A. Guagliardi, A.G.G. Moliterni, G. Polidori, J. Appl. Cryst. 28 (1995) 842.

[18] A. C. Larson, R. B. Von Dreele, General structure analysis system, Los Alamos Natl. Laboratory Report LAUR 86-748, 1994.

[19] Details were as follows: the background was modeled using a Chebyschev polynomial function with 14 terms, the default
GSAS profile function for constant wavelength X-ray data was used, and two overall isotropic atomic displacement parameters, one for mercury and one for bromine, were included in the refinement. Texture effects were taken into account using a March-Dollase texture model. Contamination by the gasket was modelled by incorporating tungsten as an additional phase.

[20] H.R. Oswald, H. Jaggi, Helv. Chim. Acta 43 (1960) 72. B. Brehler, Z. Kristallogr. 115 (1961) 373. P.H. Fourcroy, D. Carré, J. Rivet, Acta Cryst. B34 (1978) 3160. C. Chieh, M.A. White, Z. Kristallogr. 166 (1984) 189. 